\title{
Lower incisor dentoalveolar compensation and symphysis dimensions in class II and class III patients
}

\author{
Maryam Maniyar, Ajit Kalia, Ashwith Hegde, Raja Ganesh Gautam, Nasim Mirdehghan
}

\author{
Department of Orthodontics, M A Rangoonwala Dental College, Pune, Maharashtra, India
}

\begin{abstract}
Aim: The aim was to study and compare lower incisor dentoalveolar compensation and mandibular symphysis morphology of patients with Class II and Class III malocclusion. Materials and Methods: Lower incisor inclination (incisor mandibular plane angle [IMPA]), as well as buccal (LA) and lingual (LP) cortex depth, and mandibular symphysis height (LH) were measured in 60 lateral cephalometric X-rays of adult patients without prior orthodontic treatment. The subjects were divided into three groups based on antero-posterior skeletal malocclusions - Class I (control group), Class II and Class III groups. Results: IMPA and symphysis dimensions showed significant differences between the sagittal malocclusion groups. In Class III subjects, the lower incisor apex was closer to the buccal cortex, therefore, value of LA was decreased and LH was increased. In Class II subjects, the lower incisor apex was near to the lingual cortex, there value of LP was reduced and LH increased. Conclusion: Narrow alveolus was observed in Class II and III subjects compared to the Class I subjects. Natural compensation elongates the symphysis and influences the lower incisor position. Thus limiting the pre-surgical decompensation and increasing the risk of damage to periodontal tissues.
\end{abstract}

Key words: Buccal cortex, lingual cortex, lower incisor apex, occlusal plane, vertical growth pattern

\section{INTRODUCTION}

It has been known for a long time that different incisor relationships exist in similar facial types. This is often an important factor in determining the prognosis of orthodontic treatment. ${ }^{[1,2]}$ Any alteration or adjustment of one part of the dentofacial complex will require a like adjustment by another part of the complex.

Holdaway (1956), was the first one who discussed a compensatory mechanism for an acceptable facial balance related to the skeletal Class II apical base. This relation is achieved by the relative tipping of the upper

\begin{tabular}{|c|c|}
\hline \multicolumn{2}{|c|}{ Access this article online } \\
\hline Publisher & \multirow[b]{2}{*}{$\begin{array}{l}\text { Website: } \\
\text { http://www.renupublishers.com }\end{array}$} \\
\hline & \\
\hline & $\begin{array}{l}\text { DOI: } \\
\text { 10.5958/2394-4196.2014.00005.3 }\end{array}$ \\
\hline
\end{tabular}

and lower incisors. ${ }^{[1]}$ Goldsman (1959), suggested that a compensatory or balancing property exists within the dentofacial complex, which preserves the overall harmony and proportions of the facial pattern. ${ }^{[4]}$ Jacobson (1974), concluded that the dentoalveolar compensation helps to camouflage the antero-posterior and vertical basal bone discrepancies in an attempt to establish normal incisor relationship. ${ }^{[4]}$

When either basal bone, whether maxilla or mandible, deviates from its expected growth pattern, the dimensions of the alveolus are altered to camouflage the antero-posterior and vertical basal bone discrepancies. The anatomic limits set by the cortical plates of the alveolus at the incisor apex may be regarded as "orthodontic walls." ${ }^{[9]}$ The question arises, as to which patients can successfully be treated with orthodontic therapy alone and which patients will also require orthognathic surgery. Apart from esthetics, the orthodontist must also consider whether sufficient orthodontic tooth movement can be accomplished to

\footnotetext{
Address for Correspondence:

Dr. Maryam Maniyar, Department of Orthodontics, M A Rangoonwala Dental College, Pune, Maharashtra, India. E-mail: maryam.maniyar87@gmail.com
}

Submission: 28 Oct 2014; Revision: 22 Nov 2014; Acceptance: 20 Dec 2014 
correct the malocclusion with minimal iatrogenic tissue loss. It is imperative in treatment planning to consider the orthodontic walls as a limit to repositioning teeth, as well as a danger zone for unfavorable sequel such as bone loss, alveolar dehiscence, gingival recession, dental mobility and other negative effects. It is the occurrence of this unfavorable sequel that defines the borderline case as "orthodontic" or "surgical-orthodontic." [7,9,17]

The aim of our present study was to carry out a simple, visualized treatment objective with cephalometric X-rays to compare the lower incisor dentoalveolar compensation and the symphysis morphology in Class II and Class III malocclusion patients. This will help us to determine if sufficient alveolar bone is present for safe movement of incisors to correct anteroposterior skeletal discrepancies.

\section{MATERIALS AND METHOD}

\section{Inclusion Criteria}

A total of 60 lateral cephalometric X-rays were collected.

- 20 X-rays each from Class I, II and III malocclusion subjects who reported to the Department of Orthodontics, M A Rangoonwala Dental College for treatment. The subjects selected were adult non-growing patients, with vertical growth pattern between 18 and 35 years of age with complete dentition who had not received prior orthodontic treatment.

\section{CLASS I, CLASS II AND III SUBJECTS}

ANB angle was used in sagittal classification of malocclusions.

Class I (control group) - $\mathrm{ANB}=2^{\circ} \pm 2^{\circ}$, with ideal overjet and overbite.

Class II - Class II Div 1 subjects were selected.

$\mathrm{ANB}=4-8^{\circ}$

Class III $-\mathrm{ANB}=\leq-1^{\circ}$ with edge-to-edge incisor relation.

\section{Vertical Facial Type}

Tweed's mandibular plane angle was used to select patients with vertical growth pattern. Patients with, Frankfort mandibular angle $\geq 28^{\circ}$ were used for the study.

\section{Exclusion Criteria}

Patients who had received prior orthodontic treatment or maxillofacial surgery, patients who were syndromic or had root resorptions, missing teeth or erosions.

\section{Cephalometric Measurements}

All the lateral cephalometric X-rays were recorded from the same digital center in order to eliminate magnification errors. The same examiner performed all tracings by hand, on lead acetate paper using a finepoint pencil $(0.5 \mathrm{~mm})$.

\section{Lower Incisor Position [Figure 1]}

The incisor mandibular plane angle (IMPA) was obtained from the angle between the long axis of the lower incisor and the mandibular plane (control group).

\section{Symphysis Dimensions [Figure 2]}

The dimensions of the mandibular symphysis were quantified following the Handelman's criteria, Angle Orthod. 1999 (control group).

LP: Bone posterior (lingual) to mandibular incisor apex. Apex of the mandibular central incisor to the limit of the lingual cortex, along a plane parallel to the occlusal plane, drawn through the apex.

LA: Bone anterior (labial) to mandibular incisor apex. Apex of the mandibular central incisor to the limit of the labial cortex, along a plane parallel to the occlusal plane, drawn through the apex.

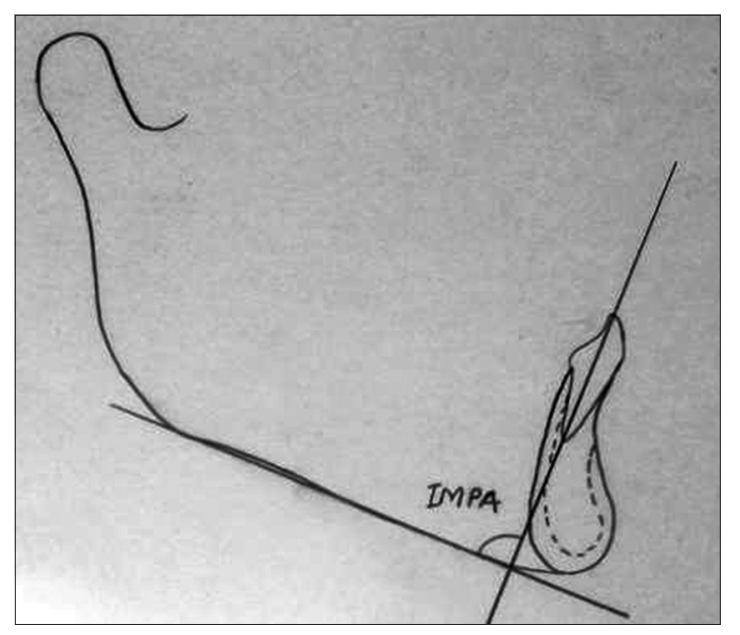

Figure 1: The incisor mandibular plane angle was obtained from the angle between the long axis of the lower incisor and the mandibular plane (Control Group) 
LH: Bone inferior to mandibular incisor apex. The shortest distance from the apex of the mandibular incisor to the lowest point of the mandibular symphysis that is transacted by a line perpendicular to the occlusal plane.

The following dimensions were measured for the Class II and Class III subjects and were compared with the control group, i.e., Class I subjects.

\section{Statistical Analysis}

Differences between the lower incisor position and symphysis dimensions were evaluated with an analysis of variance.

Simple regression analysis was used to determine the correlation coefficients between the mandibular plane, incisor position, and symphysis dimensions.

\section{RESULTS}

Table 1 and Graph 1, outlines the comparison of IMPA among the three study groups. It shows that the IMPA is increased in class II subjects and decreased in Class III subjects.

Table 2, compares the symphyseal dimensions among the three groups. And the results were summarized as follows.

\section{Class II Subjects [Figure 3]}

In Class II subjects, with proclined incisor, the apex nears the lingual cortex.

Component 1: IMPA and LA, the greater the proclination of the incisor, greater the amount of bone between the apex and the buccal cortex of the symphysis. As the value of IMPA decreases, the value of LA decreases.

Component 2: IMPA and LP, with an increase in IMPA value, the LP value decreased.

Component 3: LH, this variable determines the extrusion of the incisor. LH is increased in Class II patients as compared to the Class I subjects.

\section{Class III Subjects [Figure 4]}

In Class III subjects, with a retrolined incisor, the apex nears the buccal cortex.

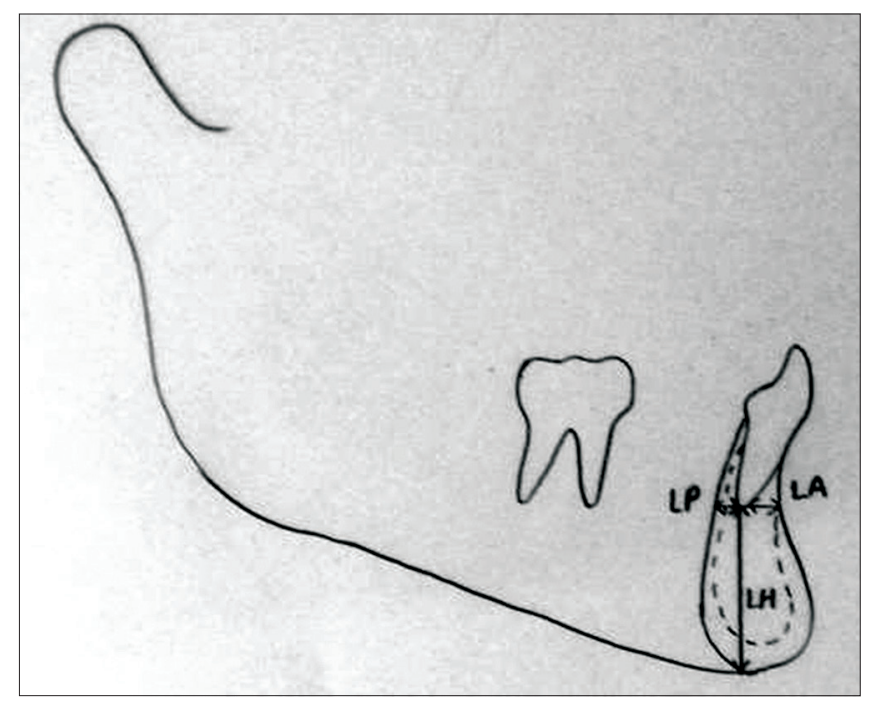

Figure 2: The dimensions of mandibular symphysis were quantified following the Handelman's criteria. (Angle Orthod. 1996) (Control Group)

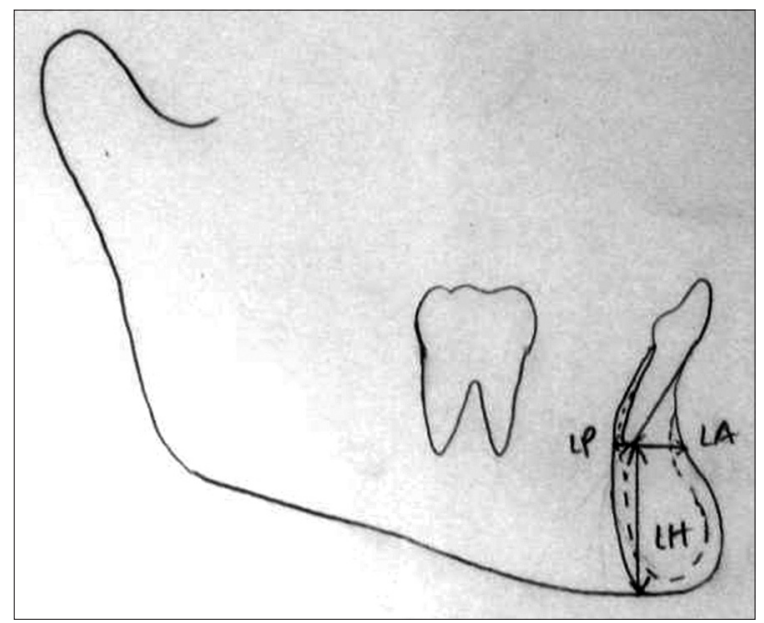

Figure 3: In Class II subjects, with proclined incisor, the apex nears the lingual cortex

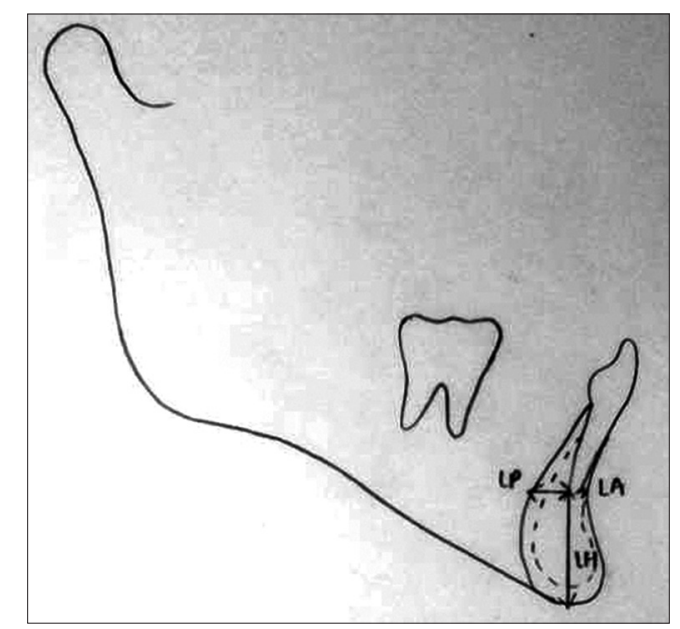

Figure 4: In Class III subjects, with a retrolined incisor, the apex nears the buccal cortex 
Table 1: The comparison of angular measurement (IMPA) across three study groups

\begin{tabular}{lcccccc}
\hline Parameter & $\begin{array}{c}\text { Class I }(\boldsymbol{n}=\mathbf{2 0}) \\
\text { control }\end{array}$ & $\begin{array}{c}\text { Class II } \\
(\boldsymbol{n}=\mathbf{2 0})\end{array}$ & $\begin{array}{c}\text { Class III } \\
(\boldsymbol{n}=\mathbf{2 0})\end{array}$ & Class I versus Class II & Class I versus Class III & Class II versus Class III \\
\hline IMPA (Deg) & $91.1 \pm 2.1$ & $106.5 \pm 2.2$ & $83.6 \pm 1.3$ & 0.001 & 0.001 & 0.001 \\
\hline $\begin{array}{l}\text { Values are mean } \pm \text { standard deviation. } \\
\text { considered to be statues by one-way analysis of variance (ANOVA) with Bonferroni's correction for multiple group comparisons. } P<0.05 \text { is }\end{array}$ \\
\hline
\end{tabular}

Table 2: The comparison of symphyseal dimensions across three study groups

\begin{tabular}{lcccccc}
\hline Parameters & $\begin{array}{c}\text { Class I }(\boldsymbol{n = 2 0}) \\
\text { control }\end{array}$ & $\begin{array}{c}\text { Class II } \\
(\boldsymbol{n}=\mathbf{2 0})\end{array}$ & $\begin{array}{c}\text { Class III } \\
(\boldsymbol{n}=\mathbf{2 0})\end{array}$ & \multicolumn{3}{c}{$\boldsymbol{P}$ values (inter group comparison) } \\
\cline { 5 - 7 } & $19.3 \pm 0.3$ & $22.9 \pm 0.6$ & $22.2 \pm 0.3$ & 0.001 & 0.001 & 0.001 \\
LH $(\mathrm{mm})$ & $3.3 \pm 0.3$ & $2.2 \pm 0.2$ & $4.6 \pm 0.3$ & 0.001 & 0.001 & 0.001 \\
LP $(\mathrm{mm})$ & $3.9 \pm 0.2$ & $4.9 \pm 0.2$ & $2.8 \pm 0.2$ & 0.001 & 0.001 & 0.001 \\
LA $(\mathrm{mm})$ & &
\end{tabular}

Values are mean \pm standard deviation. $P$ values by one-way analysis of variance (ANOVA) with Bonferroni's correction for multiple group comparisons. $P<0.05$ is considered to be statistically significant

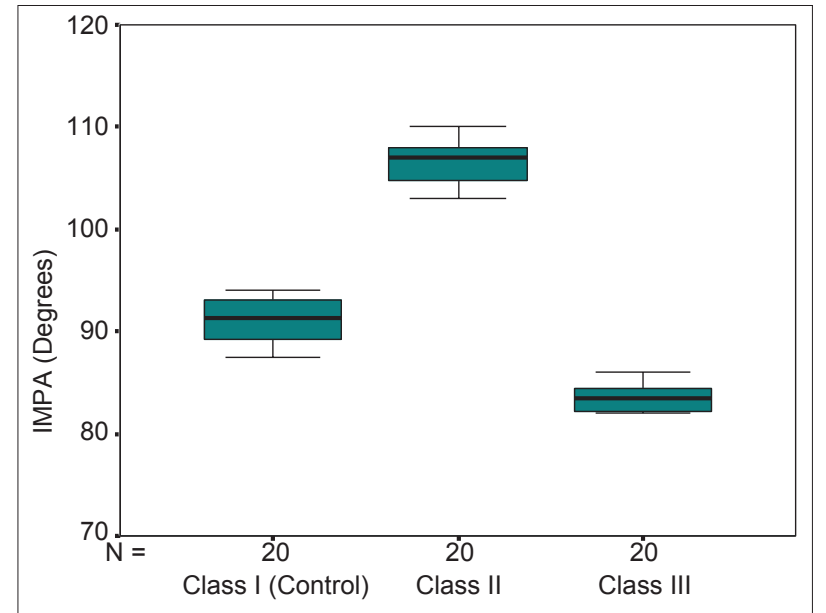

Graph 1: The comparison of angular measurement across three study groups (box-plot), in Class III subjects, incisor mandibular plane angle (IMPA) values were reduced and in Class II subjects IMPA values were increased

Component 1: IMPA and LA, greater the retroclination of the incisor, lesser the amount of bone between the apex and the buccal cortex of the symphysis.

Component 2: IMPA and LP, with a decrease of IMPA, the LP value increased.

Component 3: LH was increased in Class III patients as compared to Class I subjects.

\section{DISCUSSION}

In the present study, a comparison between Class I, Class II and Class III sample group was done in order to evaluate the dentoalveolar compensation of the lower incisor and the concomitant changes in the mandibular symphysis.

Handelman evaluated Class I, II, and III skeletal malocclusion. A decrease in the alveolar width was noted in all patients with malocclusions and long facial patterns and in Class III patients with normal faces. However, when dividing the subjects into nine subgroups, the sample sizes were small and heterogeneous ${ }^{[9]}$ Outside of Handelman's study, there are a few other studies that had explored the relationship between the alveolar bone and the behavior of the incisors as related to vertical and sagittal skeletal patterns. ${ }^{[10-13]}$

Many studies have earlier reported that mandibular prognathism or skeletal Class III malocclusion is characterized by compensatory inclinations of the maxillary and mandibular incisors for skeletal imbalances between the jaws. ${ }^{[19,23]}$ Lee et al., evaluated the alveolar bone loss around lower incisors incurred during surgical orthodontic treatment in individuals with mandibular prognathism and concluded that excessive forward movement of lower incisors during pre-surgical orthodontic treatment could cause alveolar bone loss. ${ }^{[14]}$

In prior related publications, Class III subjects were evaluated separately, but the comparison among Class II and Class III subjects with Class I subjects were not done.

In previous studies, statistical analyses sought differences between different malocclusion types but did not concurrently evaluate to what extent the symphysis dimensions were related to variations in the position of the incisor and the skeletal pattern.

\section{Clinical Significance}

At times, the skeletal discrepancy is large and beyond the "envelope of discrepancy" for orthodontic treatment. At times, this discrepancy is small but the thinness of the alveolar housing will not accommodate even modest 
movements. This is more frequently observed in the lower incisor. The dimensions of the alveolus guide us to determine how far the incisor can be translated in order to achieve the necessary correction. The incisors may be moved beyond their "envelope of discrepancy," resulting in bone loss, dehiscence, periodontal damage and other iatrogenic effects. Therefore, an assessment of the symphysis dimension helps to translate the tooth within the limit of the alveolar walls.

\section{Limitations}

Various researchers are currently studying how the morphology of the alveolar bone is affected by lower incisor proclination through three-dimensional (3D) cone-beam computed tomography. ${ }^{[15,24]}$ There is no doubt that our results will have to be compared in the future with data obtained from 3D imaging.

\section{CONCLUSIONS}

1. Cephalometric measurements were established for Class I, Class II and Class III subjects for the width of the bone lingual and buccal to the incisor apices

2. In Class III groups, extruded and retroclined incisor is often found, which causes LA to decrease, LP and LH to increase

3. In Class II groups, extruded and proclined incisor is often found, which causes LP to decrease, LA and LH to increase.

\section{REFERENCES}

1. Holdaway RA. Changes in relationship of points A and B during orthodontic treatment. Am J Orthod 1955;41:407.

2. Steiner CC. Cephalometrics for you and me. Am J Orthod 1953;39:729-55.

3. Tweed $\mathrm{CH}$. The Frankfort-mandibular plane angle in orthodontic diagnosis, classification, treatment planning, and prognosis. Am J Orthod Oral Surg 1946;32:175-230.

4. Jacobson A. The Wits appraisal of jaw disharmony. Am J Orthod 1975;67:125-38.

5. Goldsman S. The variations in skeletal and denture patterns in excellent adult facial types. Angle Orthod 1959;29:63-92.

6. Ricketts RM. Cephalometric analysis and synthesis. Angle Orthod 1961;31:141-56.

7. Bibby RE. Incisor relationships in different skeletofacial patterns. Angle Orthod 1980;50:41-4.
8. Wylie WL. The mandibular incisor - Its role in facial esthetics. Angle Orthod 1955;25:32-41.

9. Handelman CS. The anterior alveolus: its importance in limiting orthodontic treatment and its influence on the occurrence of iatrogenic sequelae. Angle Orthod 1996;66:95-109.

10. Chung CJ, Jung S, Baik HS. Morphological characteristics of the symphyseal region in adult skeletal Class III crossbite and openbite malocclusions. Angle Orthod 2008;78:38-43.

11. Troy BA, Shanker S, Fields HW, Vig K, Johnston W. Comparison of incisor inclination in patients with Class III malocclusion treated with orthognathic surgery or orthodontic camouflage. Am J Orthod Dentofacial Orthop 2009;135:146.e1-9.

12. Ishikawa $H$, Nakamura S, Iwasaki $H$, Kitazawa S, Tsukada H, Sato Y. Dentoalveolar compensation related to variations in sagittal jaw relationships. Angle Orthod 1999;69:534-8.

13. Tang N, Zhao ZH, Liao CH, Zhao MY. Morphological characteristics of mandibular symphysis in adult skeletal class II and class III malocclusions with abnormal vertical skeletal patterns. Hua Xi Kou Qiang Yi Xue Za Zhi 2010;28:395-8.

14. Lee KM, Kim YI, Park SB, Son WS. Alveolar bone loss around lower incisors during surgical orthodontic treatment in mandibular prognathism. Angle Orthod 2012;82:637-44.

15. Kook YA, Kim G, Kim Y. Comparison of alveolar bone loss around incisors in normal occlusion samples and surgical skeletal class III patients. Angle Orthod 2012;82:645-52.

16. Ten Hoeve A, Mulie RM. The effect of antero-postero incisor repositioning on the palatal cortex as studied with laminagraphy. J Clin Orthod 1976;10:804-22.

17. Solow B. The dentoalveolar compensatory mechanism: background and clinical implications. Br J Orthod 1980;7:145-61.

18. Anwar N, Fida M. Compensation for vertical dysplasia and its clinical application. Eur J Orthod 2009;31:516-22.

19. Ishikawa $H$, Nakamura $S$, Iwasaki $H$, Kitazawa S, Tsukada $H$, $\mathrm{Chu} \mathrm{S}$. Dentoalveolar compensation in negative overjet cases. Angle Orthod 2000;70:145-8.

20. Behrents RG. Growth in the Aging Craniofacial Skeleton. Monograph 17 Craniofacial Growth Series. Ann Arbor, Michigan: Center for Human Growth and Development, The University of Michigan; 1985.

21. Bishara SE, Fahl JA, Peterson LC. Longitudinal changes in the ANB angle and Wits appraisal: clinical implications. Am J Orthod 1983;84:133-9.

22. Lin J, Gu Y. Preliminary investigation of nonsurgical treatment of severe skeletal Class III malocclusion in the permanent dentition. Angle Orthod 2003;73:401-10.

23. Yamada C, Kitai N, Kakimoto N, Murakami S, Furukawa S, Takada K. Spatial relationships between the mandibular central incisor and associated alveolar bone in adults with mandibular prognathism. Angle Orthod 2007;77:766-72.

24. Yu Q, Pan XG, Ji GP, Shen G. The association between lower incisal inclination and morphology of the supporting alveolar bone - a cone-beam CT study. Int J Oral Sci 2009;1:217-23.

How to cite this article: Maniyar M, Kalia A, Hegde A, Gautam RG, Mirdehghan N. Lower incisor dentoalveolar compensation and symphysis dimensions in class II and class III patients. Int J Dent Med Spec 2014;1(2):20-24.

Source of Support: None; Conflict of Interest: None 